\title{
The long tedious journey of cleft lip and palate patients - seeks multidisciplinary approach to smoothen.
}

\author{
Md. Nazmul Hasan ${ }^{\mathbf{a}}$, Shirin Sultana Chowdhury ${ }^{\mathbf{b}}$
}

Every three minutes, somewhere in the world, a baby is born with a cleft of the lip and/or palate $(\mathrm{CL} / \mathrm{P})$, making it one of the most common birth defects ${ }^{1}$. Cleft Lip and Palate is severe birth defect occur one in 700-1000 newborn infants. As this birth defect hamper the growth, aesthetics, appearances, speech, the overall physical and psychosocial development and well being, so these patients often required generosity, guidance and keen supervision of multiple specialist health professionals. This supervision and monitoring may extended from birth to adulthood even life-longer. For the parents this longer caring period to their beloved baby born with cleft lip and palate may often not be as pleasant and enjoyable as for the normal one. Even for the patients these longer physical and psychosocial stresses are often unbearable, that could be made more improved and silky by closed supervision by interdisciplinary team approach.

The team should consist of a core group of clinicians whose predominant pediatric practice comprises the care of children with cleft lip and palate $^{2}$. These clinicians should review all children with clefts at regular, defined intervals at multidisciplinary clinics:

Specialist cleft nurse: this specialist nurse practitioner role has oversight of the integrated care of the patient and is responsible for initial antenatal contact and support, general feeding advice, social and paediatric screening and perioperative care and follow up.

Plastic Surgeon: International best practice recommends that a cleft surgeon should treat around 40-50 new cases per year in order to maintain the necessary skills and be able to audit their results.

Speech Pathologist: Speech pathologists are responsible for specialist feeding diagnosis and assessment in the early stages of development; and with growth and development, early diagnosis and assessment of speech and language development, and in some cases, delivery of treatment and oversight of community treatment regimes. Specialist clinicians with experience and expertise in instrumental speech evaluation are critical to the provision of good cleft care and the development of adequate speech and language skills for social integration.

ENT/audiology: Children with clefts are at increased risk of hearing deficits which require early identification and intervention for optimization of speech and language development in this at risk population.

Oral \& Maxillofacial Surgeons: The care of infants and adults with clefts is a highly specialized area of maxillofacial surgery, and surgical treatment into early adulthood must be planned in conjunction with dentists and orthodontic specialists.

Paediatrician: A paediatrician with specialist expertise in cleft care is necessary to provide assessment and treatment of children with complex needs - around 50\% of children with clefts will have other abnormalities, and this group is recognized as at risk for delays in development and social integration difficulties

Paediatric Dentist: Children with cleft conditions have higher risk of dental caries due to associated dental anomalies such as enamel hypoplasia and require timely regular dental review and preventive care. A paediatric dentist with experience in management of developmental anomalies of the primary and early permanent dentition is essential for regular review of oral health status.

Oral health therapist: An oral health therapist will assist the paediatric dentist in the delivery of preventive care for the child with a cleft condition.

Orthodontist: The orthodontic management of individuals with $\mathrm{CL} / \mathrm{P}$ is a recognised 
subspecialist area of orthodontics. Assessment and intervention is required at critical stages in the development of the dental occlusion, such as in the early mixed dentition, pre-alveolar bone grafting and in the early permanent dentition prior to future prosthodontic rehabilitation. In addition the provision of presurgical orthopaedics for infants with wide clefts of the lip and palate requires a high degree of skill and expertise.

Psychologist: Addressing the psychosocial needs of children with a facial difference is paramount in the successful treatment of their clefting condition. Early identification and management of such issues as social isolation, bullying, depression - all prevalent conditions in children with clefts - is the responsibility of the team with an understanding the details of treatment plans and outcomes, and such knowledge will inform treatment decisions made in consultations with patients and families.

Regarding dental care of cleft lip and palate patients, it is clear that a child with a cleft lip/palate requires the same regular preventive and restorative care as the child without a cleft. However, since children with clefts may have special problems related to missing, malformed, or mal-positioned teeth, they require early evaluation by a dentist who is familiar with the needs of the child with a cleft $^{2,3}$.

Early Dental Care: With proper care, children born with a cleft lip and/or palate can have healthy teeth. This requires proper cleaning, good nutrition, and fluoride treatment. Appropriate cleaning with a small, soft-bristled toothbrush should begin as soon as teeth erupt. Oral hygiene instructions and preventative counseling can be provided by a pediatric dentist or a general dentist. Many dentists recommend that the first dental visit be scheduled at about one year of age or even earlier if there are special dental problems. The early evaluation is usually provided through the Cleft Palate Team. Routine dental care with a local dentist begins at about three years of age. The treatment recommended depends upon many factors. Some children require only preventative care while others will need fillings or removal of a tooth.
Orthodontic Care: The first orthodontic evaluation may be scheduled even before the child has any teeth. The purpose of this visit is to assess facial growth, particularly the growth of the jaws. Later as teeth begin to erupt, the orthodontist will make plans for the child's short and long-term dental needs. For example, if a child's upper teeth do not fit together (occlude) properly with the lower teeth, the orthodontist may suggest an early period of treatment to correct the relationship of the upper jaw to the lower jaw. It is not unusual for this initial period of treatment to be followed by a long rest period when the orthodontist monitors facial growth and dental development. With the eruption of the permanent teeth, the final phase of orthodontics completes alignment of the teeth.

\section{Coordinated Dental-Surgical Care:}

Coordination of treatment between the surgeon and dental specialist is important since several procedures may be completed during the same anesthesia. Restorations or dental extractions can be scheduled at the same time as other surgery.

Coordination between the surgeon and the orthodontist becomes most important in the management of the bony defect in the upper jaw that may result from the cleft. Reconstruction of the cleft defect may be accomplished with a bone graft performed by the surgeon. The orthodontist may place an appliance on the teeth of the upper jaw to prepare for the bone graft. A retainer is usually placed after the bone graft until full braces are applied.

When the child approaches adolescence the orthodontist and the surgeon again coordinate their efforts if the teeth do not meet properly because the jaws are in abnormal positions. If the tooth relations cannot be made normal by orthodontics alone, a combined approach of both orthodontics and surgical repositioning of the jaws is necessary. Such surgery is usually performed after the pubertal growth spurt is completed.

Prosthodontic Care: The maxillofacial prosthodontist is a dental specialist who makes artificial teeth and dental appliances to improve the appearance of individuals with cleft and to 
meet their functional requirements for eating and speaking. The prosthodontist may make a dental bridge to replace missing teeth. Oral appliances called "speech bulbs" or "palatal lifts" may help close the nose from the mouth so that speech will sound more normal. The prosthodontist must also coordinate treatment with the surgeon and/or the orthodontist to assure the best possible result. When a speech bulb or palatal lift is developed, the prosthodontist usually coordinates treatment with the speech pathologist. For the child or adult who wears one of these appliances, the care of the teeth holding the appliance is of particular importance.

All those above specialist involvement can be integrated by establishing one-stop craniofacial center where those specialists will only care for cleft patients. Cleft patients are considered patients of the craniofacial center for life. Any issues with regard to clefts are managed through the clinic. Every attempt is made to achieve good continuity of care and adequate follow-up. Some realities of life can interfere, however, with consistency in care. Families may move to other parts of the country, and their care needs to be followed up by another center. Physicians as well may move, and a patient's care may be transferred to another specialist. Thorough record keeping is paramount. A patient's detailed history should be able to be reviewed in its entirety as a story of the patient's experience navigating the road of cleft care. Thus the longer tedious journey of cleft patients can be silky smoothen by team approach.

$\overline{\text { Md. Nazmul Hasan }}{ }^{a}$, Shirin Sultana Chowdhury ${ }^{b}$

a. Assistant professor and Head, Department of Orthodontics \& Dentofacial Orthopedics, Update Dental College \& Hospital, Dhaka.

b. Associated professor, Department of Conservative Dentistry and Endo-dontics, Update Dental College \& Hospital, Dhaka.

\section{Referances:}

1. Vallino-Napoli LD, Riley MM, Halliday J. An epidemiologic study of isolated cleft lip, palate, or both in Victoria, Australia from 1983 to 2000. Cleft Palate-Craniofacial Journal. 2004; 41: 185-94.

2. Albery E H, Hathorn I S, Pigott R W. Cleft lip and palate: a Team approach. Bristol: Wright, 1986.
3. Bessell A, Hooper L, Shaw WC, Reilly S, Reid J, Glenny AM. Feeding interventions for growth and development in infants with cleft lip, cleft palate or cleft lip and palate (Review). Cochrane Database of Systematic Reviews. 2011. 